\title{
On a Family of Newton-Like Methods for Bilateral Approximation of the Eigenvalues of Nonlinear Spectral Problems
}

\section{Bohdan Podlevskyi}

Pidstryhach Institute of Applied Problems for Mechanics and Mathematics of National Academy of Sciences of Ukraine, Ivan FrankoNational University of Lviv, Ukraine.

\begin{tabular}{|c|c|}
\hline ARTICLE INFO & ABSTRACT \\
\hline Published Online: & Methods and algorithms that form a one-parameter family of methods of bilateral approximations \\
\hline 20 August 2020 & to the eigenvalues of nonlinear spectral problems are constructed. Their convergence is proved. \\
\hline Corresponding Author: & Numerical results are given. \\
\hline Bohdan Podlevskyi & \\
\hline
\end{tabular}

\section{INTERODUCTION}

Nonlinear eigenvalue problems arise in various scientific and technical fields. Over the last years, significant achievements have been made in the development of numerical methods for addressing such problems. Additional information on possible applications of nonlinear eigenvalue problems and numerical methods for solving them can be obtained, in particular, from [1].

For the problems which only have real eigenvalues it would be desirable to have not only a simple approximation (for example, monotony at one side) and an asymptotic error of calculation, but also obtain the upper and lower bounds of eigenvalues in the calculation process. In many cases this allows to evaluate the reliability of the iterative approximation. It is meaning at each step of the iterative process a convenient aposteriori estimation of the calculation error can be obtained.

\section{PRELIMINARIES}

We consider the nonlinear eigenvalue problem

$$
\mathbf{D}(\lambda) y=0 \text {, }
$$

where $\mathbf{D}(\lambda)$ is a square matrix of order $n$, all elements of which are sufficiently smooth (at least three times continuously differentiable) functions of the parameter $\lambda \in R, y \in R^{n}$. The eigenvalues is sought as solutions of determinant equation

$$
f(\lambda) \equiv \operatorname{det} \mathbf{D}(\lambda)=0 \text {. }
$$

To determine the isolated eigenvalue of matrix $\mathbf{D}(\lambda)$ we proposed and justify the Newton-type iterative processes which give the alternate approximations to the eigenvalue $\lambda^{*}$ of the equation (2), i.e.

$$
\lambda_{0}<\lambda_{2}<\ldots<\lambda_{2 m}<\ldots<\lambda^{*}<\ldots<\lambda_{2 m-1}<\ldots<\lambda_{3}<\lambda_{1}
$$


or

$$
\lambda_{1}<\lambda_{3}<\ldots<\lambda_{2 m-1}<\ldots<\lambda^{*}<\ldots<\lambda_{2 m}<\ldots<\lambda_{2}<\lambda_{0}
$$

and the included monotonous bilateral approximations to the eigenvalue, i.e.

$$
\mu_{0}<\mu_{1}<\ldots<\mu_{2 m}<\ldots<\lambda^{*}<\ldots<v_{2 m}<\ldots<v_{1}<v_{0}
$$

without unfolding the determinant $\operatorname{det} \mathbf{D}(\lambda)$. This means that the left hand side of equation (2) is not set in explicit form, but the algorithm of finding the functions $f(\lambda)$ and their derivatives $f^{\prime}(\lambda)$ and $f^{\prime \prime}(\lambda)$ at a fixed value of the parameter. Therefore, for calculation $f\left(\lambda_{m}\right), f^{\prime}\left(\lambda_{m}\right)$ and $f^{\prime \prime}\left(\lambda_{m}\right)$ it is necessary compute, for a fixed $\lambda=\lambda_{m}$, decompositions

$$
\begin{aligned}
& \mathbf{D}=\mathbf{L U}, \\
& \mathbf{B}=\mathbf{M U}+\mathbf{L V}, \\
& \mathbf{C}=\mathbf{N U}+\mathbf{2 M V}+\mathbf{L W},
\end{aligned}
$$

whence we obtain

$$
\begin{gathered}
f\left(\lambda_{m}\right)=\prod_{i=1}^{n} u_{i i}, \quad f^{\prime}\left(\lambda_{m}\right)=\sum_{k=1}^{n} v_{k k} \prod_{i=1, i \neq k}^{n} u_{i i} \\
f^{\prime \prime}\left(\lambda_{m}\right)=\sum_{k=1}^{n} w_{k k} \prod_{i=1, i \neq k}^{n} u_{i i}+\sum_{k=1}^{n} v_{k k}\left(\sum_{j=1, j \neq k}^{n} v_{j j} \prod_{i=1, i \neq k, i \neq j}^{n} u_{i i}\right) .
\end{gathered}
$$

The matrix elements in the decompositions (5) can be calculated using the corresponding recurrence relations written in [3]. Consequently, in order to calculate the derivatives in $N$ points $\lambda=\lambda_{m}, m=1,2, . ., N$ it is necessary to calculate $N$ times the decomposition (6) and derivatives for each fixed $\lambda=\lambda_{m}, m=1,2, . ., N$ using formulas (6).

Next, by $\lambda^{*}$ we denote a precise simple root of equation (2) $\left(f\left(\lambda^{*}\right)=0\right)$, in some neighborhood of which the following behavior of function $f(\lambda)$ is possible.

(A). Function $f(\lambda)$ is convex $\left(f^{\prime \prime}(\lambda)>0\right)$ and its derivative is $f^{\prime}(\lambda)<0$.

(B). Function $f(\lambda)$ is concave $\left(f^{\prime \prime}(\lambda)<0\right)$ and its derivative is $f^{\prime}(\lambda)<0$.

(C). Function $f(\lambda)$ is convex $\left(f^{\prime \prime}(\lambda)>0\right)$ and its derivative is $f^{\prime}(\lambda)>0$.

(D). Function $f(\lambda)$ is concave $\left(f^{\prime \prime}(\lambda)<0\right)$ and its derivative is $f^{\prime}(\lambda)>0$.

Along with $f(\lambda)$ we consider one-parametric family of functions

$$
z_{\alpha}(\lambda)=-\frac{f(\lambda)}{\operatorname{sgn} f^{\prime} \cdot\left|f^{\prime}(\lambda)\right|^{\alpha}}, \quad \alpha \geq 0,
$$

which obviously has the same zeros as the function $f(\lambda)$, that is $z_{\alpha, \beta}\left(\lambda^{*}\right)=0$. It is easy to verify that $z_{\alpha, \beta}(\lambda)$ is twice continuously differentiable at the point of $\lambda^{*}$ for which the relation

is satisfied and which has the following properties.

$$
z_{\alpha}^{\prime}\left(\lambda^{*}\right)=-\frac{1}{\left|f^{\prime}\left(\lambda^{*}\right)\right|^{\alpha-1}}, \quad z_{\alpha}^{\prime \prime}\left(\lambda^{*}\right)=\frac{(2 \alpha-1) \cdot \operatorname{sgn} f^{\prime} \cdot f^{\prime \prime}\left(\lambda^{*}\right)}{\left|f^{\prime}\left(\lambda^{*}\right)\right|^{\alpha}} .
$$

Theorem 1. Let $\lambda^{*}$ be the simple real root of equation (2), in some neighborhood $U$ of which for function $f(\lambda)$ holds one of conditions (A) - (D). Then there is such neighborhood $U_{\varepsilon} \subset U$ of the root, in which for $\alpha \geq 1$ :

i) if conditions $(A)$ or $(D)$ is satisfied then the function $z_{\alpha}(\lambda)=-f(\lambda) / \operatorname{sgn} f^{\prime} \cdot\left|f^{\prime}(\lambda)\right|^{\alpha}$ is a concave monotonically decreasing function, its derivative $z_{\alpha}^{\prime}(\lambda)<0$ and monotonically decreasing;

ii) if conditions (B) or (C) is satisfied then the function $z_{\alpha}(\lambda)=-f(\lambda) / \operatorname{sgn} f^{\prime} \cdot\left|f^{\prime}(\lambda)\right|^{\alpha}$ is a convex monotonically decreasing function, its derivative $z_{\alpha}^{\prime}(\lambda)<0$ and monotonically increasing. 


\section{Problems"}

\section{A FAMILY OF BILATERAL APPROXIMATION METHODS}

Using the properties of the function $z_{\alpha}(\lambda)$, we can construct a family of iterative processes of Newton type methods that give bilateral approximations in the form (3). For example, for cases (A) or (D), the iterative process can be written in the form

$$
\left\{\begin{array}{l}
\lambda_{2 m+1}=\lambda_{2 m}-\frac{f\left(\lambda_{2 m}\right)}{f^{\prime}\left(\lambda_{2 m}\right)\left|f^{\prime}\left(\lambda_{2 m}\right)\right|^{\alpha-1}}, \\
\lambda_{2 m+2}=\lambda_{2 m+1}-\frac{f\left(\lambda_{2 m+1}\right) f^{\prime}\left(\lambda_{2 m+1}\right)}{f^{\prime}\left(\lambda_{2 m+1}\right)^{2}-\alpha f\left(\lambda_{2 m+1}\right) f^{\prime \prime}\left(\lambda_{2 m+1}\right)},
\end{array}\right.
$$

$m=0,1,2, \ldots, \quad \lambda_{0} \in\left(\lambda^{*}, \lambda^{*}+\varepsilon\right)$,

Similarly, an iterative process can be constructed for other cases.

The following theorem serves to justify the bilateral convergence of the iterative process (7).

Theorem 2. Let $\lambda^{*}$ be a simple real root of equation (2) and let us suppose that in some neighborhood of the root $U_{\varepsilon}\left(\lambda^{*}\right)=\left\{\lambda:\left|\lambda-\lambda^{*}\right|<\varepsilon\right\}$,

where the following relation fulfills

$$
\left|\alpha \frac{f(\lambda) f^{\prime \prime}(\lambda)}{f^{\prime}(\lambda)^{2}}\right| \leq q<1
$$

for a three times continuously differentiable function $f(\lambda)$ that describes the equation (2) the condition $(A)$ or $(D)$ is satisfied, and for the function $z_{\alpha}(\lambda)=-f(\lambda) / \operatorname{sgn} f^{\prime} \cdot\left|f^{\prime}(\lambda)\right|^{\alpha}$ the inequalities

$$
\begin{array}{ll}
-\frac{1}{z_{\alpha}^{\prime}\left(\lambda_{0}\right)}>N & \text { for } \quad \lambda_{0} \in\left(\lambda^{*}, \lambda^{*}+\varepsilon\right), \\
\left|\frac{z_{\alpha}\left(\lambda_{1}\right)}{z_{\alpha}^{\prime}\left(\lambda_{1}\right)}\right|<\frac{2}{M} \quad \text { for } \quad \lambda_{1} \in\left(\lambda^{*}-\varepsilon, \lambda^{*}\right),
\end{array}
$$

are hold, where

$$
M=\max _{\lambda \in U_{\varepsilon}\left(\lambda^{*}\right)}\left|z_{\alpha}^{\prime \prime}(\lambda)\right|, \quad N=\max _{\lambda \in\left(\lambda^{*}-\varepsilon, \lambda^{*}\right)}\left|\frac{z_{\alpha}(\lambda) z_{\alpha}^{\prime \prime}(\lambda)}{z_{\alpha}^{\prime}(\lambda)^{2}}\right|<1 .
$$

Then the iterative process (7) starting with $\lambda_{0} \in\left(\lambda^{*}, \lambda^{*}+\varepsilon\right)$ converges to the root $\lambda^{*}$ on both sides

$$
\lambda_{1}<\lambda_{3}<\ldots<\lambda_{2 m-1}<\ldots<\lambda^{*}<\ldots<\lambda_{2 m}<\ldots<\lambda_{2}<\lambda_{0} .
$$

The theorem is proved by the method of mathematical induction, relying on Theorem 1 .

It should be noted that an iterative process (7), which provides the bilateral approximations to the eigenvalue, with respect to (6), will be presented in the form

$$
\begin{gathered}
\lambda_{2 m+1}=\lambda_{2 m}-1 /\left(\sum_{k=1}^{n} \frac{\bar{v}_{k k}}{\bar{u}_{k k}}\right) \cdot\left|\sum_{k=1}^{n} \frac{\bar{v}_{k k}}{\bar{u}_{k k}}\right|^{\alpha-1} \cdot\left|\prod_{i=1}^{n} \bar{u}_{i i}\right|^{\alpha-1}, \\
\lambda_{2 m+2}=\lambda_{2 m+1}-\left(\sum_{k=1}^{n} \frac{v_{k k}}{u_{k k}}\right) / \sum_{k=1}^{n}\left(\left(\frac{v_{k k}}{u_{k k}}\right)^{2}-\alpha \frac{w_{k k}}{u_{k k}}+(1-\alpha) \frac{v_{k k}}{u_{k k}}\left(\sum_{i=1, i \neq k}^{n} \frac{v_{i i}}{u_{i i}}\right)\right),
\end{gathered}
$$

$m=0,1,2, \ldots$

where $\bar{u}_{k k}, \bar{v}_{k k}$ are the elements of the matrices $\mathbf{U}, \mathbf{V}$ in the decompositions (5) for the fixed $\lambda=\lambda_{2 m}$, and $u_{k k}, v_{k k}, w_{k k}$ are the elements of the matrices $\mathbf{U}, \mathbf{V}$ and $\mathbf{W}$ in the decompositions (5) for the fixed $\lambda=\lambda_{2 m+1}$.

So, the algorithm can be written as:

Algorithm 1. Iterative process of alternate approximations

1. We set the initial approximation $\lambda_{0}$ to the $s$-th eigenvalue of the problem (1)

2. for $m=0,1,2, \ldots$ until the accuracy is achieved do 


\section{Problems"}

3. if $m$ is even

4. than calculate the values $\bar{u}_{k k}, \bar{v}_{k k}$ from the decomposition (5) for $\lambda=\lambda_{2 m}$

5. calculate approximation to the eigenvalue $\lambda_{2 m+1}$ by (8)

6. else calculate the values $u_{k k}, v_{k k}, w_{k k}$ from the decomposition (5) for $\lambda=\lambda_{2 m+1}$

7. calculate approximation to the eigenvalue $\lambda_{2 m+2}$ by (8)

8. end for $m$.

The algorithm shows that in order to obtain the alternate approximations, on each step of the algorithm, one must refer to the algorithm of calculating the decomposition (5).

In some cases, the algorithm constructed based on the iterative process of including approximations is more optimal with respect to the number of calls to the calculation of decomposition (5) [3]:

$$
\begin{aligned}
& \mu_{m+1}=\mu_{m}-\frac{f\left(\mu_{m}\right)}{f^{\prime}\left(\mu_{m}\right)}, \\
& v_{m+1}=\mu_{m}-\frac{f\left(\mu_{m}\right) f^{\prime}\left(\mu_{m}\right)}{f^{\prime}\left(\mu_{m}\right)^{2}-\alpha f\left(\mu_{m}\right) f^{\prime \prime}\left(\mu_{m}\right)}, \quad m=0,1,2, \ldots .
\end{aligned}
$$

With this algorithm we get the including approximations to the eigenvalue in the form $\lambda_{0}=\mu_{0}<\mu_{1}<\ldots<\mu_{m}<\ldots<\lambda_{*}<\ldots<v_{m}<\ldots<v_{2}<v_{1}$,

using one initial approximation, for example, to the left hand of $\lambda_{*}$.

If we now replace the values of the function and its derivatives at the desired points by the relations (A.4), then the iterative process (9) will have the form

$$
\begin{gathered}
v_{m+1}=\mu_{m}-1 /\left(\sum_{k=1}^{n} \frac{v_{k k}}{u_{k k}}\right) \\
\lambda_{2 m+2}=\lambda_{2 m+1}-\left(\sum_{k=1}^{n} \frac{v_{k k}}{u_{k k}}\right) / \sum_{k=1}^{n}\left(\left(\frac{v_{k k}}{u_{k k}}\right)^{2}-\alpha \frac{w_{k k}}{u_{k k}}+(1-\alpha) \frac{v_{k k}}{u_{k k}}\left(\sum_{i=1, i \neq k}^{n} \frac{v_{i i}}{u_{i i}}\right)\right),
\end{gathered}
$$

$m=0,1,2, \ldots$

where $u_{k k}, v_{k k}, w_{k k}$ are the elements of the matrices $\mathbf{U}, \mathbf{V}$ and $\mathbf{W}$ in the decompositions (5) for the fixed $\lambda=\mu_{m}$.

Therefore, we propose the following algorithm to find the eigenvalues of the nonlinear spectral problem:

Algorithm 2. Iterative process of included approximations

1. We set the initial approximation $\lambda_{0}=\mu_{0}$ to the s-th eigenvalue of the problem (1)

2. for $m=0,1,2, \ldots$ until the accuracy is achieved do

3. calculate the values $u_{k k}, v_{k k}, w_{k k}$ from the decomposition (5) at the $\lambda=\mu_{m}$

4. calculate approximations to the eigenvalue $\mu_{m+1}$ and $\nu_{m+1}$ by the formula (10)

5. end for $m$.

Thus, we see that with the algorithm 2, unlike the algorithm 1, two approximations (from left and right hand sides of the root) are calculated performing only one call to the calculation of decomposition (5).

\section{NUMERICAL RESULTS}

In the study of the stability of systems of ordinary differential equations with delay, the eigenvalue problems in which the spectral parameter enters nonlinearly are arisen. Thus, the process of propagation of weak perturbations in multi-connected systems (such as electric and acoustic fields) is described by a system of linear differential-algebraic equations of the form

$$
\mathbf{B} \frac{d u(t)}{d t}+\mathbf{A} u(t)+\sum_{k=1}^{N} \mathbf{C}_{k} u\left(t-\tau_{k}\right)=0
$$


where $\mathbf{A}, \mathbf{B}, \mathbf{C}_{\mathbf{k}}$ are the square matrix, $\tau_{k}>0, k=1,2, \ldots, N$ are the given numbers. When investigation of the stability consider that the elements of the matrices $\mathbf{A}, \mathbf{B}, \mathbf{C}_{\mathbf{k}}$ do not depend on $t$ and on the delay parameters $\tau_{1}, \tau_{2}, \ldots, \tau_{N}$.

We consider the partial case of such system with one delay parameter and also assume that the coefficients of the equations and the parameter are independent of the time variable. Then the system of equations will take the form

$$
\mathbf{B} \frac{d u(t)}{d t}+\mathbf{A} u(t)+\mathbf{C} u(t-\tau)=0
$$

where $u(t)$ is the vector of perturbations, $\mathbf{A}, \mathbf{B}, \mathbf{C}$ are square real matrices whose coefficients are independent of $t$. Then the solutions of system (12) can be found in the form

$$
u(t)=x e^{\lambda t}
$$

where $x$ is the desired vector, $\lambda$ is the number. Substituting (13) into system (12), we obtain the eigenvalue problem

$$
\left(\mathbf{A}+\lambda \mathbf{B}+e^{-\tau \lambda} \mathbf{C}\right) x=0 .
$$

Since (14) is a homogeneous system of linear algebraic equations, then its non-trivial solution exists if and only if

$$
\operatorname{det}\left(\mathbf{A}+\lambda \mathbf{B}+e^{-\tau \lambda} \mathbf{C}\right)=0 .
$$

Next, we consider problem (14) in which the matrix $\mathbf{B}=\mathbf{C}=\mathbf{E}$. This choice of the problem is dictated by the fact that for it it is possible to compare the results of work of the proposed algorithms $1-2$ with some other algorithms that were tested on a such problem.

Therefore, we consider a nonlinear spectral problem $\mathbf{D}(\lambda) x=0$ with matrix

$$
\mathbf{D}(\lambda)=\mathbf{A}+\lambda \mathbf{E}+e^{-\lambda} \mathbf{E},
$$

where $\mathbf{E}$ is a unit matrix, $\mathbf{A}$ is a tridiagonal matrix with nonzero elements: $a_{i-1, i}=a_{i, i+1}=1, a_{i i}=-2$.

For such matrices, all the eigenvalues of problem (15) were calculated by each of algorithms $1-2$. They completely coincided with the numbers obtained in [5] by another algorithm, which is only applicable if the matrices in problem (14) are related by the relation $\mathbf{C}=\alpha \mathbf{B}$. The results of the numerical experiments for the three eigenvalues are given in Table. 1. The calculations were performed with accuracy $\varepsilon=10^{-6}$.

Table 1. Bilateral Approximation

\begin{tabular}{|c|c|c|c|c|}
\hline & & Algorithm 1 & \multicolumn{2}{|c|}{ Algorithm 2 } \\
\cline { 3 - 5 }$\lambda_{0}$ & $\boldsymbol{m}$ & $\lambda_{m}$ & $\boldsymbol{\mu}_{m}$ & $\boldsymbol{v}_{m}$ \\
\hline 4.0 & 1 & 3.941742 & 3.843018 & 3.941742 \\
& 2 & 3.885413 & 3.885413 & 3.909833 \\
& 3 & 3.900572 & 3.897677 & 3.899646 \\
& 4 & 3.898688 & 3.898710 & 3.898726 \\
& 5 & 3.898718 & 3.898718 & 3.898718 \\
& 6 & 3.898718 & - & - \\
\hline 3.4 & 1 & 3.324599 & 3.242099 & 3.324599 \\
& 2 & 3.271814 & 3.270297 & 3.272956 \\
& 3 & 3.271783 & 3.271782 & 3.271784 \\
& 4 & 3.272783 & 3.271783 & 3.271783 \\
\hline 0.5 & 1 & 0.620603 & 0.620603 & 0.663037 \\
& 2 & 0.644214 & 0.643877 & 0.644074 \\
& 3 & 0.643963 & 0.643963 & 0.643963 \\
& 4 & 0.643963 & - & - \\
\hline
\end{tabular}

\section{CONCLUSIONS}

Approbation of the constructed algorithms on model and physical problems shows their reliability and efficiency, as well as advantages over the usual Newton's method in the sense that at each step of the iterative process we obtain bilateral estimates of the desired solution, and therefore at each step we get a convenient a posteriori estimation of calculation error.

As for the choice of the $\alpha$ parameter, this is the subject of a separate study. Here we note only next. When $\alpha=1 / 2$ is selected, the convergence rate increases and becomes cubic. When $\alpha=1$, we can obtain the rate of convergence not less than cubic, using algorithm 2 , in which for each subsequent 
approximation to take the middle of the interval, ie $\mu_{k+1}=\left(\mu_{k}+v_{k}\right) / 2$.

Partial cases of this family of methods have been used to find the points of branching solutions of nonlinear integral equations arising in the theory of the synthesis of radiating systems and are used in the design of radiating devices (different types of antenna systems),

\section{REFERENCES}

1. Mehrmann V., Voss H. Nonlinear Eigenvalue Problems: A Challenge for Modern Eigenvalue Methods. GAMM Mitteilungen, 2004: 27: 121152. https://doi.org/10.1002/gamm.201490007

2. Podlevs'kyi B. M. Bilateral Analog of the Newton Method for Determination of Eigenvalues of Nonlinear Spectral Problems. J. Math. Sci. 2009:
160: 357-367. https://doi.org/10.1007/s10958-0099503-2.

3. Podlevskyi B. M. Bilateral Methods for Solving of Nonlinear Spectral Problems, Nauk dumka, Kyiv, 2014. (in Ukrainian).

4. Podlevskyi B. One Approach to Construction of Bilateral Approximations Methods for Solution of Nonlinear Eigenvalue Problems. American Journal of Computational Mathematics. 2012:2: 118-124. https://doi.org/10.4236/ajcm.2012.22016.

5. Kartyshov S. V. Numerical method for solving the eigenvalue problem for sparse matrices depending non-linearly on a spectral parameter. Comput. Math. Math. Phys. 1989: 29: 209-213. https://doi.org/10.1016/S0041-5553(89)80032-1 